\title{
L'ARCHITECTURE (DURABLE) COMME TECHNOLOGIE DE GOUVERNEMENT : APPORTS ET DÉTOURNEMENTS DE LA SOCIOLOGIE DE L'ACTION PUBLIQUE
}

\author{
Julie Neuwels
}

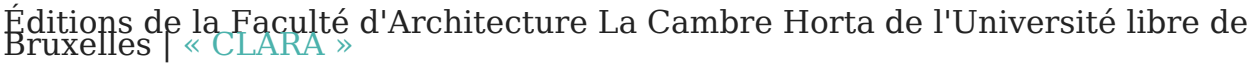

2015/1 N 3 | pages 63 à 72

ISSN 2295-3671

ISBN 9782804702588

Article disponible en ligne à l'adresse :

https://www.cairn.info/revue-clara-2015-1-page-63.htm

Distribution électronique Cairn.info pour Éditions de la Faculté d'Architecture La Cambre Horta de l'Université libre de Bruxelles.

(C) Éditions de la Faculté d'Architecture La Cambre Horta de l'Université libre de Bruxelles. Tous droits réservés pour tous pays.

La reproduction ou représentation de cet article, notamment par photocopie, n'est autorisée que dans les limites des conditions générales d'utilisation du site ou, le cas échéant, des conditions générales de la licence souscrite par votre établissement. Toute autre reproduction ou représentation, en tout ou partie, sous quelque forme et de quelque manière que ce soit, est interdite sauf accord préalable et écrit de l'éditeur, en dehors des cas prévus par la législation en vigueur en France. Il est précisé que son stockage dans une base de données est également interdit. 


\section{L'ARCHITECTURE (DURABLE) COMME TECHNOLOGIE

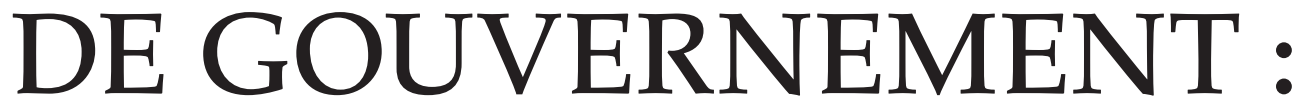

APPORTS ET DÉTOURNEMENTS DE LA SOCIOLOGIE DE L'ACTION PUBLIQUE

\section{Julie Neuwels}

La transition de l'habiter vers un " développement durable » fait l'objet $d^{\prime}$ 'une attention particulière par les sociologues. Certains d'entre eux s'inscrivent dans le courant dit cognitiviste de la sociologie de l'action publique. Se situant entre les sciences politiques et la sociologie, ce courant de recherche insiste sur les dimensions cognitives de la construction des problèmes publics, soit les influences et les évolutions des représentations qui sont faites du réel. En ce sens, ce courant permet de questionner la notion de «durable » sous l'angle des transformations de notre conception du « développement». Il offre ainsi des prises pour se distancer du registre performatif qui domine actuellement le champ des recherches s'attachant à l'architecture durable tant dans le registre de l'ingénierie, par la définition quantitative des enjeux et de leurs solutions, que dans le registre de la sociologie, par l'analyse de l'appropriation par les acteurs en situation des évolutions techniques et normatives.

Cette capacité à dépasser la question performative nous a conduite à prendre appui sur ce pan de la sociologie. Cependant, dans les approches classiques, l'enjeu scientifique consiste en l'étude des évolutions des modalités de l'action justifiées au nom du durable. La ville et l'architecture constituent alors des terrains d'étude. A contrario, nos recherches s'attachent à l'architecture et non à l'action publique. 
Notre angle de lecture est donc inversé : il ne s'agit pas de questionner les mutations de l'action justifiées au nom du développement durable à travers l'analyse de la production architecturale, mais bien de questionner les mutations de l'architecture justifiées au nom du développement durable par l'analyse de l'action publique. Cette inversion de l'angle de lecture implique une série de distanciations vis-à-vis de la sociologie de l'action publique, tout en orientant la manière dont la question architecturale est abordée par la mise en exergue de ses dimensions politiques et cognitives.

Cet article vise à souligner la nature de cette rencontre entre architecture et sociologie cognitiviste de l'action publique. Cette rencontre sera mise à l'épreuve par l'analyse de l'« appel à projets bâtiments exemplaires ", ellemême abordée à travers les modalités de construction de l'intérêt général. Au-delà des enseignements quant à la rencontre traitée, ce cas d'étude illustre certaines évolutions du rôle sociétal alloué à l'architecture, de ses usages politiques et des particularités du contexte bruxellois à ces égards.

\section{LE « DURABLE », UNE QUESTION SOCIOCOGNITIVE}

La notion de développement durable arbore une portée critique en ce qu'elle appelle à revoir l'ensemble des constructions sociocognitives de notre rapport au développement : notre conception du progrès, de la nature, des techniques, de la science, de l'humain, de l'économie, etc. Par extension, le domaine de l'habiter n'échappe pas à ces remises en question structurelles qui ne peuvent être limitées à des considérations performatives (Pinson et al., 2011).

Le développement durable n'est ni une signification stabilisée uniformément partagée, ni un outil opératoire définissant précisément des objectifs et des moyens. Les appréhensions du degré transformationnel de sa portée critique s'avèrent donc pour le moins diversifiées, jusqu'à relever de l'asymétrie entre approches conciliatoires (croissance économique, approche utilitariste de la nature, confiance envers le progrès technique, etc.) et approches réformistes (décroissance, lenteur, écologie profonde, etc.) (Godard, 2004 ; Bourg et Fragnière, 2014). De plus en plus d'auteurs considèrent ainsi que les débats ne portent plus sur la nécessité de considérer les enjeux environnementaux, mais bien sur les évolutions des constructions sociocognitives, ou des " référentiels » dans la sémantique de la sociologie de l'action publique (Hajer, 1995 ; Rumpala, 2003).

Dans la lignée des travaux de Michel Foucault, les référentiels sont définis comme étant les représentations qui sont faites d'un problème et $d^{\prime}$ 'un secteur d'action, et sur la base desquelles reposent la construction de l'action publique, ses objectifs et ses dispositions (Muller, 2000). Bien que demeurant de l'ordre de l'interprétation jusqu'à parfois relever du 
paralogisme, ces représentations se stabilisent en tout ou en partie. Elles s'imposent alors comme un modèle naturel d'interprétation de la réalité dont il est difficile de se défaire, à l'image du référentiel de marché caractérisé par l’idée que la croissance économique mène au progrès.

Ces cadres théoriques influencent la manière dont nous questionnons l'architecture durable. Tout d'abord, dans la lignée de la conception du développement durable sous l'angle de sa portée critique, les mutations de l'architecture sont analysées dans ses relations avec les représentations faites du développement. Il s'agit de mettre en évidence comment la notion d'architecture durable est appropriée, et les évolutions cognitives qui en résultent. Ensuite, l'importance prêtée aux aspects cognitifs nous amène à considérer l'architecture comme étant en elle-même une construction sociocognitive évoluant en fonction des référentiels globaux conformément à $l^{\prime}$ " imaginaire architectonique " conceptualisé par Antoine Picon (2001 : 21).

L'ensemble de ces influences relève d'une appréhension de l'architecture comme une «technologie de gouvernement " telle que définie par Michel Foucault : un concept relevant plus d'une construction culturelle que d'un phénomène naturel, présenté comme une réalité qui s'impose, telle la folie, la sexualité ou la société civile, en vue de légitimer une politique et de servir d'interface entre les gouvernants et les gouvernés (Foucault, 2004). Selon cette logique, l'architecture durable est abordée comme « un champ de référence nouveau, une réalité nouvelle sur quoi s'exerce l'art de gouverner » (ibid. : 299).

\section{UN RETOUR À L'HYGIÉNISME ?}

Les approches cognitivistes s'intéressant au développement durable ne cherchent pas à analyser le degré de véracité ou d'efficacité de l'action, mais bien son degré de changement ainsi que la signification de ce changement. L'enjeu méthodologique ne relève alors pas de l'analyse du référentiel du développement durable en tant que tel, mais de l'analyse de ses traductions opérationnelles. À cet égard, certains auteurs utilisent la transposition du durable dans le domaine de l'habiter comme terrain d'étude, car la ville, le quartier et le projet architectural constituent des unités d'analyse circonscrites et des espaces privilégiés d'application de politiques environnementales (Theys, 2002 ; Pinson et al., 2011).

Cherchant à questionner les évolutions des modalités de l'action induites par le durable, le rapport aux acteurs dans la construction de l'intérêt général y est couramment abordé. Deux grandes conceptions de l'action peuvent être distinguées à cet égard (Lascoumes et Le Bourhis, 1998). La première, normative et basée sur des savoirs exclusivement " experts ", 
consiste en la définition unilatérale des problèmes et des solutions qu'il s'agit d'imposer aux acteurs en situation. La seconde, plus pragmatique, car intégrant des savoirs dits " profanes", consiste en la construction collective, évolutive et située de l'intérêt général.

Les analyses sociologiques s'intéressant à cette question mettent en évidence que la transposition du durable dans le domaine de l'habiter s'effectue à travers un renforcement de l'approche normative conformément au concept d' ' éco-pouvoir » défini par Pierre Lascoumes comme étant la « reprise en main par les experts scientifiques et techniques des décisions essentielles en matière de gestion des milieux, d'administration des risques, et de construction sociale des problèmes d'environnement " (1994 : 286). À l'échelle de l'action, cette confiance techniciste renforce les approches en termes d'acceptabilité sociale des solutions proposées par les experts, au détriment de la réévaluation collective de notre rapport au développement (Rumpala, 2003).

Plus qu'une "valorisation de l'évidence de l'ingénierie écologique " (Villalba, 2011 : 56), l'éco-pouvoir s'accompagne d'une " expansion de l'activité étatique " caractérisée par une charge morale importante (Rumpala, 2003 : 354). Celle-ci se formalise, entre autres, par divers dispositifs de sensibilisation et d'éducation des citoyens : guides édictant des manières d'habiter plus respectueuses de l'environnement, suivis des consommations énergétiques dans certains logements sociaux et écoquartiers, ou encore équipements techniques censés influencer les comportements et les représentations des citoyens. Ces instruments témoignent d'une «inquiétude liée à la mise à l'épreuve de la ville durable par ceux qui l'habitent ", à la résistance aux changements sous-tendus par ses équipements et logiques (Boissonade, 2010).

Cet idéal techniciste et sa charge morale augmentent le clivage entre efficacité et légitimité en exacerbant le rôle des experts et la méfiance envers les présupposées inerties des comportements et attitudes individualistes (Salles, 2006 ; Ferguson, 2013). Certains auteurs y voient ainsi un retour des logiques hygiénistes (Reigner, 2012; Renauld, 2012). Ce retour révèle un échec de certaines transformations de l'action prêtées au durable : maintien de la confiance accordée aux techniques et aux experts, manque de considération pour l'incertitude et le complexe ainsi que pour les savoirs profanes.

Ces constats, ici fortement résumés, ont servi de supports pour mettre en évidence les particularités du cas bruxellois et pour problématiser les évolutions du rapport à l'architecture qui en résultent. L'« appel à projets bâtiments exemplaires " est représentatif de ces apports. Il témoigne que le cas bruxellois se démarque de prime abord du concept d'éco-pouvoir et de son pendant hygiéniste, mais qu'en même temps, cette distanciation s'avère fragile. 


\section{L'APPEL À PROJETS BÂTIMENTS EXEMPLAIRES, UNE APPROCHE CRÉATIVE}

Mis en place en 2007, cet instrument est un appel à projets annuel assimilable à une labellisation, mais très proche du concours d'architecture. Les projets soumis sont analysés individuellement par un jury d'experts. Éloignées des diktats normatifs, seules quatre grandes orientations sont définies : la minimisation des impacts écologiques, la qualité architecturale, l'intégration au contexte et la reproductibilité technique et financière. Bien qu'invités à respecter le standard passif en construction neuve et basse énergie en rénovation, les projets peuvent y déroger si cela s'avère opportun.

Par un contrat signé avec Bruxelles Environnement ${ }^{1}$ et par diverses modalités de suivi, les porteurs des projets s'engagent à respecter les caractéristiques du ou des bâtiments lauréats qu'ils ont eux-mêmes définies. En retour, dans une visée d'incitation et de facilitation, ils bénéficient d'une importante publicité, de primes financières et d'encadrements techniques.

La démarche est pragmatique. Elle laisse percevoir que les acteurs de terrain ne sont pas considérés comme individualistes, mais comme des individualités capables de défendre le bien commun et porteuses de solutions essentielles à considérer pour construire une projection commune de ce que pourrait être une architecture durable. L'enjeu politique consiste à leur offrir des prises pour inciter un questionnement et faciliter l'engagement.

1

Administration de la Région de Bruxelles-Capitale en charge de l'exécution de diverses matières en lien avec l'environnement (air, eau, bruit, espaces verts, énergie, etc.).

\section{2}

Au sujet du projet $L^{\prime} E s p o i r$, voir le blog. Consultable : http://espoirmolenbeek. blogspot.be [disponible le 24 novembre 2014].
Cette mise en questionnement a ainsi favorisé et/ou mis en évidence le développement de solutions variées entendant répondre à divers aspects soulevés par la notion de développement durable. Ces solutions abordent en partie les aspects environnementaux de la question architecturale. À l'échelle des enjeux énergétiques notamment, les bâtiments exemplaires témoignent d'une grande diversité en matière de construction passive par rapport aux autres villes européennes en termes d'expression architecturale (formes et parements), de taille des projets (de l'extension de la maison unifamiliale au plus grand bâtiment tertiaire passif d'Europe), de méthodes constructives (matériaux et équipements variés, construction neuve et rénovation) et de programmation (tertiaire, crèche, école, mosquée, etc.). Mais les solutions proposées intègrent également des approches alternatives, voire innovantes, du point de vue socio-économique. Les projets de logements collectifs Brutopia et L'Espoir sont régulièrement cités à cet égard : le premier témoignant des avantages de l'autopromotion et de l'habitat groupé, le second ayant permis l'accession à la propriété privée à des familles défavorisées grâce à des processus solidaires et participatifs ${ }^{2}$. 
À l'opposé de l'éco-pouvoir, l'architecture est donc appréhendée comme un espace de construction collective et située de l'intérêt général. L'architecture durable n'est pas perçue comme un enjeu uniquement performatif, mais également comme un espace de réflexion et de création, rejoignant la conception du développement durable comme une mise en questionnement structurelle de nos rapports à l'habiter. Ces caractéristiques font de l'appel à projets un instrument singulier, innovant. Elles témoignent d'une approche "créative » des politiques publiques qui, laissant la place à l'expérimentation, s'inscrit dans les logiques d'action généralement accolées au durable (Bourdin, 2012).

\section{L'USAGE POLITIQUE DES BÂTIMENTS EXEMPLAIRES, UNE FRAGILISATION DE L'APPROCHE CRÉATIVE}

Cet éloignement avec l'éco-pouvoir n'est cependant valable qu'à l'échelle de l'appel à projets à proprement parler. De fait, les usages politiques des bâtiments labellisés exemplaires, réalisés ou non, en fragilisent la caractéristique créative. Notre intérêt pour les usages politiques qui sont faits des bâtiments introduit une divergence méthodologique avec la sociologie de l'action publique, car si le projet urbain y est reconnu comme étant une modalité d'action publique (Pinson, 2004), l'architecture y demeure appréhendée comme un simple réceptacle pour la mise en œuvre et à l'épreuve des politiques (agir sur l'architecture), et non comme étant également un instrument à part entière (agir par l'architecture). Le cas de l'appel à projets bâtiments exemplaires illustre pourtant l'influence de l'utilisation de l'architecture comme instrument de régulation sur la construction du référentiel d'architecture durable.

Les usages politiques des bâtiments labellisés exemplaires s'avèrent multiples. En premier lieu, les bâtiments sont utilisés dans une optique pédagogique visant les acteurs non engagés dans la démarche. Pour ce faire, la visibilité des projets et leur caractère démonstratif constituent des critères de sélection clairement énoncés dans les appels à projets, et sont assurés par la suite par des panneaux de chantier spécifiques, des plaques signalétiques situées à côté des portes d'entrée, l'organisation de visites, de séminaires et diverses publications. Cet usage témoigne d'une recherche d'influence des comportements et représentations par la spatialité. Dans le cadre des bâtiments exemplaires, elle se formalise par un nouveau rapport à la matérialité architecturale qui se doit, par son esthétique, d'exprimer et de renforcer le renouveau du secteur et l'effectivité des politiques en œuvre.

Ensuite, à l'échelle plus globale de la construction du référentiel d'architecture durable à Bruxelles, les bâtiments exemplaires constituent un support à la justification de l'imposition du presque passif entrée en 
vigueur en janvier $2015^{3}$. Ils sont présentés, d'une part comme formant une «masse critique de projets » suffisante pour en assurer la pertinence et la faisabilité, et d'autre part comme témoignant que les bâtiments passifs relèvent $d$ ' " attentes des maîtres d'ouvrage et du grand public ». Dans les discours, la confusion entre les bâtiments exemplaires et l'imposition du presque passif est importante. Ces presque deux cents projets sont notamment présentés sur le site Internet dédié à l'imposition ${ }^{4}$ sans distinction de leur performance énergétique ou de l'effectivité de leur réalisation ${ }^{5}$.

Parallèlement, parce qu'ils constituent un « outil de distinction " (Béal, 2013 : 219), les bâtiments exemplaires sont enrôlés dans la politique d'attractivité de la Région. Entre autres, ils font l'objet de visites de délégations étrangères et ont constitué un pan majeur des dossiers de candidature de Bruxelles pour les Sustainable Energy Europe Awards (lauréate en 2012 dans la catégorie Living), l'Earth Hour City Challenge (lauréate en 2014) ou encore pour la désignation de la Capitale verte de l'Europe 2015 (finaliste).

3

Législation régissant la performance énergétique de certains bâtiments neufs et rénovations lourdes construites sur la base de la certification passive tout en prévoyant des mesures de facilitation, à savoir le report de l'imposition des exigences d'étanchéité à l'air en 2018, le libre choix du système de ventilation et la mise en place d'un système d'exception permettant une dérogation automatique.

4

Consultable : http://www. brusselpassief.be [disponible le 29 septembre 2014].

5

Divers bâtiments exemplaires sont (très) basse énergie. Quelques projets ont été abandonnés.
Cette intégration dans les politiques de régulation coercitive et d'attractivité influence l'usage de ces bâtiments, mis en scène pour créer du désir pour le durable et pour construire l'image d'une supposée révolution verte de la ville. Les retours d'expériences relèvent dès lors d'un récit idéalisé faisant principalement état des aspects positifs. Ce récit est constructif en donnant à penser et en favorisant la mobilisation (Villalba, 2009). De fait, les bâtiments exemplaires ont participé à la popularisation de l'écologisation de l'architecture. Mais ce récit influence l'appréhension des problématiques. Les quelques aléas évoqués sont généralement incombés aux mauvais usages des bâtiments et au manque de formation des praticiens. Tandis que les attitudes sceptiques, voire contestataires, face à l'imposition du presque passif, sont considérées comme résultant d'une inertie au changement, d'un manque d'information et/ou d'un individualisme.

La construction du récit idéalisé repose donc sur une double exacerbation : du caractère idéal de certaines pratiques, mais également de l'incrimination de ceux qui n'en respectent pas les normes. Soit un renforcement du clivage entre les "bons » acteurs et les autres qu'il faut encore responsabiliser, entre les bâtiments " exemplaires " dont il faut s'inspirer et les autres qui n'ont rien à apprendre au collectif. Ce clivage renforce la concentration des efforts sur l'acceptabilité sociale des solutions avancées au détriment de l'attention pour l'adéquation desdites solutions et pour la signification des comportements incriminés 
(Shove, 2003). En ce sens, de par les usages secondaires qui sont faits des bâtiments exemplaires, l'appel à projets rejoint les critiques évoquées précédemment, sans pour autant tomber dans une exacerbation hygiéniste.

Ces évolutions incombent moins à l'instrument en tant que tel qu'à son inscription dans les logiques de réglementation juridique et de marketing territorial. Les registres au sein desquels les bâtiments exemplaires sont utilisés illustrent et influencent simultanément les représentations qui sont faites tant de l'architecture que du développement durable. L'architecture tend à être moins perçue comme un espace réflexif que comme un champ de pratiques problématiques pour lequel il existe des solutions à appliquer immédiatement au nom d'un intérêt général. Cela sous-tend une conception du développement durable comme étant un objectif défini au détriment de sa portée critique et, donc, réflexive.

\section{CONCLUSION}

L'appel à projets et les bâtiments exemplaires illustrent les apports et limites de la rencontre entre le pendant cognitiviste de la sociologie de l'action publique et l'architecture. Nous en retiendrons ici trois grands aspects en guise de conclusion.

Les cadres théoriques et méthodologiques utilisés permettent d'aborder l'architecture durable sous un angle peu exploré jusqu'à présent, à savoir les évolutions du rapport politique à l'architecture induites par la référence au «durable». Cette approche permet de se distancer du registre de la performativité. Cependant, en nous conduisant à appréhender l'architecture comme une technologie de gouvernement, cette rencontre balise notre objet d'étude à son seul rôle de support à l'action. Support qui a été questionné ici en termes d'équilibre entre une conception de l'architecture durable comme étant un espace de mise en questionnement ou comme étant un espace de mise en œuvre de solutions stabilisées. L'usage de la sociologie de l'action publique ne permet donc pas d'aborder divers aspects du référentiel d'architecture durable tels que son efficacité ou ses influences esthétiques et formelles.

En mettant en exergue l'importance des dimensions cognitives, la sociologie de l'action publique offre des prises permettant de temporiser "l'empressement à théoriser le changement et les virtualités positives » du durable (Pinson et al., 2011 : 11). Elle permet ainsi d'élargir le champ des critiques, jusqu'il y a peu principalement énoncées en termes de décalage entre déclarations et actions (Béal, 2011). En même temps, en se référant au concept de référentiel, il s'avère difficile d'échapper à un positionnement critique, potentiellement contestable du point de vue scientifique. 
Enfin, la sociologie de l'action publique arbore une visée généralisante, cherchant à rendre compte des évolutions globales de l'action à partir de l'étude de cas situés. A contrario, dans les recherches portant sur l'habiter durable à proprement parler, il est généralement considéré que les tentatives de caractérisations globalisantes à partir de cas situés doivent se faire avec prudence. Le cas bruxellois présente effectivement des particularités le distinguant des autres politiques européennes. Dès lors, les résultats issus de la sociologie de l'action publique sont considérés avant tout comme un support facilitant la problématisation et ouvrant certaines pistes de réflexion, et non comme des données transposables en l'état.

\begin{abstract}
Architecte de formation, Julie Neuwels est doctorante au sein du laboratoire Architecture et Sciences humaines (Sasha) et du Centre d'études en développement, territoire et paysages (HABITER) à la Faculté d'architecture de l'Université libre de Bruxelles. Sa thèse traite des évolutions des relations entre l'architecture et le développement dans le contexte occidental, en particulier eu égard à la recherche d'un développement plus durable en Région de Bruxelles-Capitale. Julie Neuwels est également architecte praticienne, associée au sein du collectif metamorphOse.
\end{abstract}

L'auteure remercie Typhaine Moogin pour ses remarques pertinentes et constructives.

\section{BIBLIOGRAPHIE}

BÉAL,V. 2011. «Ville durable et justice sociale. Ce que le développement durable nous dit de la production de l'urbain ", dansV. Béal; M. Gauthier et G. Pinson (sous la dir. de), Le développement durable changera-t-il la ville? Le regard des sciences sociales, SaintÉtienne, Publications de l'Université de Saint-Étienne, p. 239-259.

BÉAL, V. 2013. « La dépolitisation ambiguë de l'action publique environnementale. Sélection des acteurs et transformation des rapports policy/politics dans les villes françaises et britanniques ", dans G. Le Naour; G. Massardier (sous la dir. de), L'action publique sélective, Paris, L.G.D.J., p. 205-225.

BOISSONADE, J.2010. Des épreuves critiques aux mises en justice de la ville durable. Les moments critiques transactionnels de l'écoquartier Grand Large à Dunkerque. Consultable sur le site du Réseau de recherche euro-méditerranéen développement durable et lien social : http://www.reseau-2dlis.eu/uploads/ fichiers_PDF/Boissonade_epreuves_ critiques_mise_en_justice_ville_durable ecoquatier_grand_large_Dunkerque.pdf [disponible le 19 avril 2014].
BOURDIN, A. 2012. « Le développement durable crée-t-il fatalement un urbanisme de la préservation?", dans N. Dris (sous la dir. de), Patrimoines et développement durable. Ressources, enjeux, lien social, Rennes, Presses universitaires de Rennes, p. 23-34.

BOURG, D. ; FRAGNIÈRE, B. 2014. La pensée écologique. Une anthologie, Paris, PUF.

FERGUSON,Y. 2013. «L'instrumentation de l'action publique comme mode de sélection "rationnel" de ses acteurs légitimes ", dans G. Le Naour ; G. Massardier (sous la dir. de), L'action publique sélective, Paris, L.G.D.J., p. 89-108.

FOUCAULT, M. 2004. Naissance de la biopolitique : cours au Collège de France (1978-1979), Paris, Seuil.

GODARD, O. 2004. « De la pluralité des ordres - Les problèmes d'environnement et de développement durable à la lumière de la théorie de la justification ", Géographie, économie, société, $\mathrm{n}^{\circ} 3, \mathrm{p} .303-330$.

HAJER, M. 1995. The Politics of Environmental Discourse. Ecological Modernization and the Policy Process, New York, Oxford University Press.
LASCOUMES, P.; LE BOURHIS, J.-P. 1998. "Le bien commun comme construit territorial. Identités d'action et procédures ", Politix, n 42, p.37-66.

LASCOUMES, P. 1994. L'éco-pouvoir : environnements et politiques, Paris, La Découverte.

LASCOUMES, P.; LE GALÈS, P. (sous la dir. de) 2004. Gouverner par les instruments, Paris, Presses de Science Po.

MULLER, P. 2000. «L'analyse cognitive des politiques publiques : vers une sociologie politique de l'action publique ", Revue française de science politique, $\mathrm{n}^{\circ} 2$, p. 189-208.

PICON, A. 2001. «Imaginaires de l'efficacité, pensée technique et rationalisation ", Réseaux, n 109, p. 18-50.

PINSON, G. 2004. « Le projet urbain comme instrument d'action publique ", dans P. Lascoumes ; P. Le Galès (sous la dir. de), Gouverner par les instruments, Paris, Presses de Science Po, p. 199-233.

PINSON, G. ; BÉAL, V.; GAUTHIER, M. 2011. «Introduction ", dans V. Béal ; M. Gauthier et G. Pinson (sous la dir. de), op.cit., p. 9-30.

REIGNER, H. 2012. « Usages et usagers fantasmés de la planification urbaine durable.Vers un gouvernement 


\section{L'ARCHITECTURE (DURABLE) COMME TECHNOLOGIE DE GOUVERNEMENT}

néohygiéniste des conduites? ", Colloque Sociologie des approches critiques $d u$ développement et de la ville durables, Paris.

RENAULD, V. 2012. Fabrication et usage des écoquartiers français : éléments d'analyse à partir des quartiers de Bonne (Grenoble), Ginko (Bordeaux) et BottièreChénaie (Nantes), thèse de doctorat, Lyon, INSA de Lyon.

RUMPALA, Y. 2003. Régulation publique et environnement. Questions écologiques, réponses économiques, Paris, L'Harmattan.

SALLES, D. 2006. Les défis de l'environnement: démocratie et efficacité, Paris, Syllepse.

SHOVE, Elizabeth, 2003. Comfort, Cleanliness and Convenience. The Social Organization of Normality, Oxford, Berg.

THEYS, J. 2002. « L'approche territoriale du "développement durable", condition d'une prise en compte de sa dimension sociale ", Développement durable et territoires. Consultable : http://developpementdurable.revues. org/1475 [disponible le 15 avril 2014].

VILLALBA, B. 2009. «L'utopie sociale de la ville durable ", Ecorev' Revue critique d'écologie politique. Consultable : http://ecorev.org/spip.php?article648 [disponible le 21 avril 2014].

VILLALBA, B. 2011. «La professionnalisation de la ville durable : contributions à la standardisation du développement durable ", dansV. Béal ; M. Gauthier et G. Pinson (sous la dir. de), op.cit., p.37-57. 